\title{
Cortical State Determines Global Variability and Correlations in Visual Cortex
}

\author{
Marieke L. Schölvinck, ${ }^{1}$ Aman B. Saleem, ${ }^{1}$ Andrea Benucci, ${ }^{1}{ }^{\oplus}$ Kenneth D. Harris,,${ }^{1,2}$ and ${ }^{\oplus}$ Matteo Carandini ${ }^{1}$ \\ ${ }^{1}$ UCL Institute of Ophthalmology, University College London, London EC1V 9EL, United Kingdom, and ${ }^{2}$ UCL Institute of Neurology and UCL Department \\ of Neuroscience, Physiology \& Pharmacology, London WC1E 6DE, United Kingdom
}

\begin{abstract}
The response of neurons in sensory cortex to repeated stimulus presentations is highly variable. To investigate the nature of this variability, we compared the spike activity of neurons in the primary visual cortex (V1) of cats with that of their afferents from lateral geniculate nucleus (LGN), in response to similar stimuli. We found variability to be much higher in V1 than in LGN. To investigate the sources of the additional variability, we measured the spiking activity of large V1 populations and found that much of the variability was shared across neurons: the variable portion of the responses of one neuron could be well predicted from the summed activity of the rest of the neurons. Variability thus mostly reflected global fluctuations affecting all neurons. The size and prevalence of these fluctuations, both in responses to stimuli and in ongoing activity, depended on cortical state, being larger in synchronized states than in more desynchronized states. Contrary to previous reports, these fluctuations invested the overall population, regardless of preferred orientation. The global fluctuations substantially increased variability in single neurons and correlations among pairs of neurons. Once this effect was removed, pairwise correlations were reduced and were similar regardless of cortical state. These results highlight the importance of cortical state in controlling cortical operation and can help reconcile previous studies, which differed widely in their estimate of neuronal variability and pairwise correlations.
\end{abstract}

Key words: vision; thalamus; cerebral cortex; neural populations; brain states

\section{Introduction}

The response of neurons in visual cortex to repeated stimulus presentations is highly variable. This trial-to-trial variability can be as large as the response itself (Heggelund and Albus, 1978; Tolhurst et al., 1983; Vogels et al., 1989; Carandini, 2004). However, cortical neurons are not noisy spiking machines (Mainen and Sejnowski, 1995; Deweese and Zador, 2004). The source of their variability must therefore lie in their synaptic inputs (Shadlen and Newsome, 1998; Carandini, 2004). These inputs must be at least partially shared because variability is typically correlated across neurons (Cohen and Kohn, 2011). It is essential

Received Nov. 28, 2013; revised Sept. 25, 2014; accepted 0ct. 26, 2014.

Author contributions: M.L.S., K.D.H., and M.C. designed research; M.L.S., A.B., K.D.H., and M.C. performed research; A.B. contributed unpublished reagents/analytic tools; M.L.S., A.B.S., and M.C. analyzed data; M.L.S., A.B.S., K.D.H., and M.C. wrote the paper.

This work was supported by the Wellcome Trust, the Engineering and Physical Sciences Research Council (United Kingdom), and the European Research Council. M.L.S. was supported by a Human Frontiers Science Program longterm postdoctoral fellowship. M.C. holds the GlaxoSmithKline/Fight for Sight Chair in Visual Neuroscience. We thank Tobe Freeman, Severine Durand, Daniel Kiper, Valerio Mante, and Vincent Bonin for helping acquire data with single electrodes; Steffen Katzner and Laura Busse for helping acquire data with Utah arrays; and David Schulz for spike sorting the latter data.

The authors declare no competing financial interests.

This article is freely available online through the J Neurosci Author Open Choice option.

Correspondence should be addressed to Dr. Marieke L. Schölvinck, Ernst Strüngmann Institute for Neuroscience in Cooperation with Max Planck Society, 60528 Frankfurt-am-Main, Germany. E-mail: marieke.scholvinck@esi-frankfurt.de.

A. Benucci's present address: RIKEN Brain Science Institute, Wako City 351-0198, Japan.

DOI:10.1523/JNEUROSCI.4994-13.2015

Copyright $\odot 2015$ Schölvinck et al.

This is an Open Access article distributed under the terms of the Creative Commons Attribution License (http://creativecommons.org/licenses/by/3.0), which permits unrestricted use, distribution and reproduction in any medium provided that the original work is properly attributed. to understand how variability and correlation in cortical responses arise, and what factors influence them. Indeed, correlated variability places critical constraints on information processing (Shadlen and Newsome, 1998; Ringach, 2009), and excessive variability may result in cognitive deficits (Dinstein et al., 2012).

In primary visual cortex (V1), two main views have been proposed for the synaptic origins of variability. In the first view, variability arises from addition of sensory responses onto ongoing (spontaneous) cortical activity (Arieli et al., 1996). This ongoing activity, in turn, has been proposed to resemble the patterns of activity evoked by oriented stimuli (Tsodyks et al., 1999; Kenet et al., 2003), suggesting a relationship between variability and similarity in orientation preference. The second view proposes that cortical variability is inherited from thalamic inputs, independently of cortical circuitry (Priebe and Ferster, 2012). In support of this view, Sadagopan and Ferster (2012) reported that, in many $\mathrm{V} 1$ neurons, response variability was unaffected by silencing local cortical inputs.

Distinguishing between these views has been difficult. First, it is not clear how the variability of V1 firing responses compares with that of their afferents from lateral geniculate nucleus (LGN). Multiple studies measured variability in LGN (for review, see Sadagopan and Ferster, 2012) and in V1 (for review, see Carandini, 2004), but few have directly compared the two (Kara et al., 2000; Goris et al., 2014). Generally, responses in cortex are thought to be more variable than in LGN, but the one study that concurrently measured the two (Kara et al., 2000) is difficult to interpret because its measurements in V1 indicated much less variability than in the rest of the literature. 
Second, the degree to which response variability is shared among large groups of V1 neurons is still uncertain. Experiments measuring V1 variability concentrated on single neurons (for review, see Carandini, 2004) or pairs (Cohen and Kohn, 2011), and only rarely on larger populations (e.g., Goard and Dan, 2009).

Finally, the relationship between correlated variability and cortical state is still under debate (Kohn et al., 2009). The cortex can exhibit a continuum of states (Harris and Thiele, 2011): from a synchronized state characterized by strong, low-frequency fluctuations in global activity to a desynchronized state where these fluctuations are absent (Steriade, 2006). Cortical state affects stimulus responses and their variability in multiple sensory cortices (Kisley and Gerstein, 1999; Poulet and Petersen, 2008; Curto et al., 2009; Goard and Dan, 2009; Marguet and Harris, 2011). How does it affect the correlated variability of V1 neurons and the structure of ongoing activity?

\section{Materials and Methods}

Experiments were performed at the Smith-Kettlewell Eye Research Institute following protocols approved by the local authorities. We used 8 cats for experiments with single electrodes in LGN, 7 cats for experiments with single electrodes in V1, and 9 cats for array recordings in V1. Some of the same data have appeared in studies where we focused on responses obtained after averaging across multiple presentations of each stimulus (Bonin, 2005; Durand et al., 2007; Mante et al., 2008; Nauhaus et al., 2008; Benucci et al., 2009). Here, we examine them in individual presentations.

Physiology. Young adult cats (all female) were anesthetized with sodium pentothal $(0.5-2 \mathrm{mg} / \mathrm{kg} / \mathrm{h})$ and fentanyl (typically $10 \mu \mathrm{g} / \mathrm{kg} / \mathrm{h}$ ) supplemented with inhalation of $\mathrm{N}_{2} \mathrm{O}$ mixed with $\mathrm{O}_{2}$ (typically 70:30). A neuromuscular blocker was given to prevent eye movements (pancuronium bromide, $0.15 \mathrm{mg} / \mathrm{kg} / \mathrm{h}$, i.v.). The cat was artificially respirated and received periodic doses of an antibiotic (cephazolin, $20 \mathrm{mg} / \mathrm{kg}$, twice daily), an antiedematic steroid (dexamethasone, $0.4 \mathrm{mg} / \mathrm{kg}$ daily), and an anticholinergic agent (atropine sulfate, $0.05 \mathrm{mg} / \mathrm{kg}$, i.m., daily). A craniotomy was performed over area $\mathrm{V} 1$ to insert single electrodes or electrode arrays. Temperature, EEG, heart rate, end-tidal $\mathrm{CO}_{2}$, and lung pressure were continuously monitored. Experiments typically lasted $48-96 \mathrm{~h}$.

Stimuli. Stimuli were sequences of brief stationary gratings, each flashed for $8 \mathrm{~ms}$ in LGN recordings and for $8-48 \mathrm{~ms}$ (typically, $32 \mathrm{~ms}$ ) in V1 recordings (Benucci et al., 2009). Experiments consisted of 4-6 sequences lasting 6-25 s, separated by blank intervals, each repeated in random order at least 3 times in LGN experiments, and at least 10 times in V1 experiments. The gratings were large (typically 15 degrees diameter) and varied randomly among $4-20$ orientations and 4 spatial phases. Contrast was $50 \%-80 \%$ (100\% in one experiment), and spatial frequency was optimized by a preliminary experiment (typically, 0.5 cycles/ degree). In experiments involving multielectrode arrays, randomly interleaved with the gratings were blank frames (uniform gray screen, lasting $32 \mathrm{~ms}$ ) that occurred with a probability of $\sim 33 \%$, and the sequences were interspersed with 6-10 s long blanks, to record ongoing activity.

Recordings. To compare LGN and V1 responses (see Fig. 1), we considered recordings of single-unit activity (53 neurons in LGN and 53 in V1) obtained with quartz-coated platinum/tungsten microelectrodes (Thomas Recording), which were advanced until spikes had good isolation from the background.

The rest of the recordings are from $10 \times 10$ electrode arrays (Blackrock Microsystems, $400 \mu \mathrm{m}$ spacing, $1.5 \mathrm{~mm}$ length) inserted $0.8-1 \mathrm{~mm}$ deep into V1. From each site, we obtained multiunit activity (MUA), after filtering the raw data with a high-pass filter of $250 \mathrm{~Hz}$. Spikes in MUA were detected as crossings of a threshold of $4 \mathrm{SD}$ of the background noise. In 5 experiments, we also obtained responses from well-isolated single neurons, by clustering in the space of the first three principal component coefficients, and assigning to single neurons those clusters that were well segregated from background noise (Nauhaus et al., 2008).
We computed the orientation preference of each MUA site using reverse correlation (Benucci et al., 2009) and binned sites in 12 bins of orientation preference. Sites with firing rate $<1$ spikes/s were excluded. In two experiments, we confirmed the correct assignment of orientation preference using independent data (full-field gratings presented at $100 \%$ contrast for $2 \mathrm{~s}$ ).

Variability index. To measure variability, we first counted spikes in bins of a certain width (as indicated for each analysis) and then computed a variability index (the Fano factor) as the ratio of the variance to the mean spike count. To control for differences in number of sequences or number of trials across experiments, for each experiment we only analyzed responses to one of the 4-6 stimulus sequences, and we considered every random permutation of 3 repeats for each experiment (even though in most cases we had acquired at least 10).

To characterize ongoing fluctuations in population activity, we considered MUA measured during blank screen periods. We defined a global fluctuation index by averaging across all sites and dividing the SD of this averaged activity by its mean across time.

Global noise model. To analyze the variability of data recorded in the arrays, we considered the activity of each unit (a well-isolated neuron, or the MUA of a recording site, depending on the analysis), and we computed firing rate by binning its spikes in bins of a given width (as indicated for each analysis). We then expressed the responses $R_{j k}(t)$, at each unit $j$, trial $k$, and time $t$ as the sum of a sensory signal term, $\bar{R}_{j}(t)$, obtained by averaging the responses across trials, and a noise term, $N_{j k}(t)$ as follows:

$$
R_{j k}(t)=\bar{R}_{j}(t)+N_{j k}(t)
$$

We then computed the global noise $\bar{N}_{k \neq j}(t)$ by averaging the noise terms across all the other units $k \neq j$. We used this global noise to obtain a model prediction $M_{j k}(t)$ for unit $j$, by summing the sensory signal in that unit to a weighted version of the global noise as follows:

$$
M_{j k}(t)=\bar{R}_{j}(t)+\alpha_{j} \bar{N}_{k \neq j}(t)
$$

The weights $\alpha_{j}$ were optimized by linear regression. They were set to zero when evaluating predictions based on the stimulus alone.

We defined the local noise as the difference between the measured noise $N_{j k}(t)$ and the weighted global noise $\alpha_{j} \bar{N}_{k \neq j}(t)$

To define the portion of the variance of the measured response explained by the model, we used the following formula for each unit $j$ :

$$
E_{j}=1-\operatorname{var}\left(\sum_{k} R_{j k}(t)-M_{j k}(t)\right) / \operatorname{var}\left(\sum_{k} R_{j k}(t)\right)
$$

We then averaged the explained variance over units.

Prediction quality was measured with cross-validation: weights were estimated based on a training set ( $80 \%$ of the time points) and used to explain the variance of the test set (the remaining 20\%). For consistency across experiments, we only analyzed responses to the first 10 repeats (trials) of one stimulus sequence in each experiment, regardless of how many more may have been collected.

In addition to this global noise model, for MUA we also considered a more flexible peer-prediction model, in which each site could give a different weight to each other site (Harris et al., 2003; Pillow et al., 2008; Truccolo et al., 2010). However, this model did not lead to increased cross-validated performance, so we did not adopt it.

Pairwise correlations. Using the responses on single trials, we computed the correlations $r_{i j}$ between activity $R_{i k}(t)$ and $R_{j k}(t)$ at each pair of sites $i$ and $j$. We then computed noise correlations by performing the same analysis on the residuals between responses measured in individual trials and responses averaged across trials, $N_{j k}(t)$ and $N_{i k}(t)$ (Cohen and Maunsell, 2009). To quantify how correlation depended on orientation preference similarity, we binned the pairs according to their difference in preferred orientation before averaging all correlations in a bin.

\section{Results}

To investigate neuronal variability in different cortical states, we examined activity in the visual system of anesthetized cats. We 

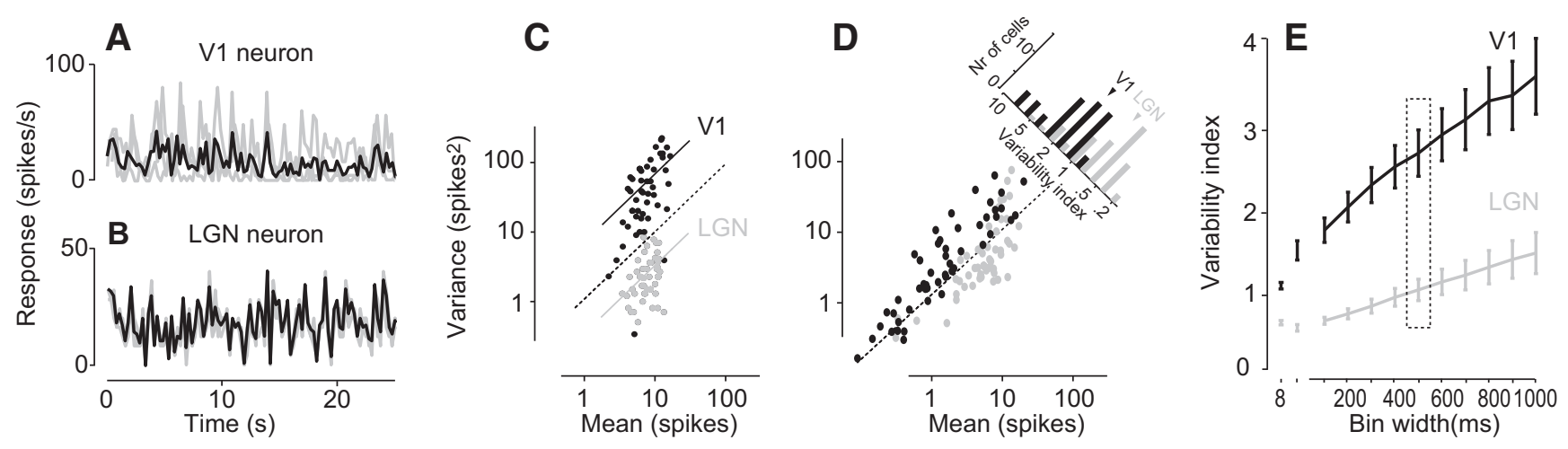

Figure 1. Response variability in LGN and V1. $\boldsymbol{A}$, The responses of a single V1 neuron to three repetitions of a $25 \mathrm{~s}$ stimulus sequence consisting of rapidly flashed gratings. Gray represents individual responses; black represents average response. Experiment 50-12-13. B, Same as in A, for an LGN neuron. Experiment 24-6-13. C, Variance of spike counts versus mean spike count for the LGN neuron (gray) and the V1 neuron (black). Spikes were counted over bins with width of $500 \mathrm{~ms}$; each individual dot represents one bin. Dotted line indicates the identity: mean equals variance. Continuous lines indicate the average ratio of variance to mean for the two neurons. $D$, Variance of spike counts versus mean spike count for all LGN (gray) and V1 (black) neurons. Top right, Histogram, Ratio of variance to mean (single-unit variability index, or Fano factor) for all V1 and LGN neurons. Error bars indicate \pm 1 SE. Arrows indicate the medians for LGN neurons and for V1 neurons. $\boldsymbol{E}$, The average variability index as a function of bin width. Ticks on the $x$-axis denote a bin width of 8-50-100 .. $1000 \mathrm{~ms}$. Rectangle represents the bin width used in $\boldsymbol{A}-\boldsymbol{C}$.

recorded from individual neurons in V1 and LGN using single electrodes (Bonin et al., 2005), and from neuronal populations in V1 using $10 \times 10$ electrode arrays (Benucci et al., 2009). Stimuli were sequences of brief stationary gratings varying rapidly in orientation and phase, interspersed randomly with several seconds of gray screen to record ongoing activity (Ringach et al., 1997).

\section{Variability in V1 greatly exceeds variability in LGN}

We began by comparing the variability of single neurons in V1 and LGN in their responses to multiple presentations of the same stimulus. The responses of a typical V1 neuron were highly variable: the firing rate traces measured in three trials were clearly different from each other and therefore from their mean (Fig. $1 A)$. Conversely, the responses of a typical LGN neuron were much more similar across trials (Fig. $1 B$ ). The difference in variability between the two neurons can be quantified by plotting the relationship between mean and variance of their spike counts during the course of the response (Fig. 1C). Although mean spike counts were similar ( $\sim 10$ spikes per $500 \mathrm{~ms}$ bin $)$, the variance of the spike counts was much higher in the V1 neuron than in the LGN neuron.

The responses of V1 neurons typically had markedly higher variance than LGN neurons (Fig. $1 D, E$ ). We considered the responses of $53 \mathrm{~V} 1$ neurons and 53 LGN neurons, and for each we calculated a single-unit variability index as the ratio between variance and mean of the spike counts (Fano factor). For most V1 neurons, the variability index was substantially higher than for LGN neurons (Fig. $1 D): 2.7 \pm 0.3$; mean \pm SE $(N=53)$ in $\mathrm{V} 1$ versus $1.0 \pm 0.1(N=53)$ in LGN. The difference in variability between LGN and V1 was evident regardless of the bin size we used to compute the variability index, indicating that the increased variability seen in V1 covers a wide range of time scales (Fig. 1E).

This difference between LGN and V1 agrees qualitatively with measures made separately for neurons in LGN (Levine and Troy, 1986; Reich et al., 1997) and in V1 (Dean, 1981; Tolhurst et al., 1983; Bradley et al., 1987; Geisler and Albrecht, 1997). Our measurements improve on this literature because we used essentially identical stimuli to measure from LGN and V1 (with a slight difference in temporal content because LGN neurons follow faster temporal frequencies than V1 neurons). Moreover, a separate analysis performed on the responses to a different type of stimulus (drifting gratings) revealed similar differences in variability between LGN and V1 (Bonin et al., 2003). Indeed, because V1 responses are so variable, we typically had to repeat each stimulus at least 10 times when recording from V1 to obtain good estimates of visual responses, compared with only 3 times when recording from LGN (this difference in number of trials does not affect the results here because it was taken into consideration, see Materials and Methods).

\section{Predicting the variability of V1 neurons}

Is the large variability of V1 responses shared across neurons? To address this question, we analyzed activity measured with a $10 \times$ 10 multielectrode array in cat V1, in response to the same kinds of stimuli (flashed gratings with random orientations). We asked whether we could predict the variability in the responses of one V1 neuron based on the activity of the remaining population. The latter was defined as the summed activity of all isolated single neurons, excluding the site of the neuron under study.

As expected, the responses of a typical V1 neuron to multiple repeats (trials) of the same sequence of gratings displayed substantial trial-to-trial variability (Fig. $2 A$, black). This activity is therefore poorly predicted by the mean sensory response (i.e., the activity averaged across repeats), which is by definition identical across trials (Fig. 2A, gray). Indeed, the variable portion of the activity (the noise) was generally large relative to the mean sensory response: the excursion in firing rates due to noise was comparable with the excursion caused by the most extreme changes in visual stimulation (Fig. $2 B$ )

Although large, the noise for a given neuron could be predicted quite precisely based on a simple model (Eq. 2): the sum of the noise at all other neurons in the array, which we term global noise, multiplied by a single scaling factor, which could be different across neurons. When we added this term to the mean sensory response shown by each neuron, it yielded a prediction that closely matched that neuron's firing rate (Fig. 2C,D). Similar results were obtained for the other neurons measured by the array (Fig. 2E) and in all 5 experiments for which we had well-isolated single neurons: the responses of a V1 neuron could be invariably predicted better when taking into account not only the sensory response but also the global noise (Fig. $2 F$, circles).

The success of the global noise model indicates that much of the variability in V1 is shared across neurons. This success did not 
A
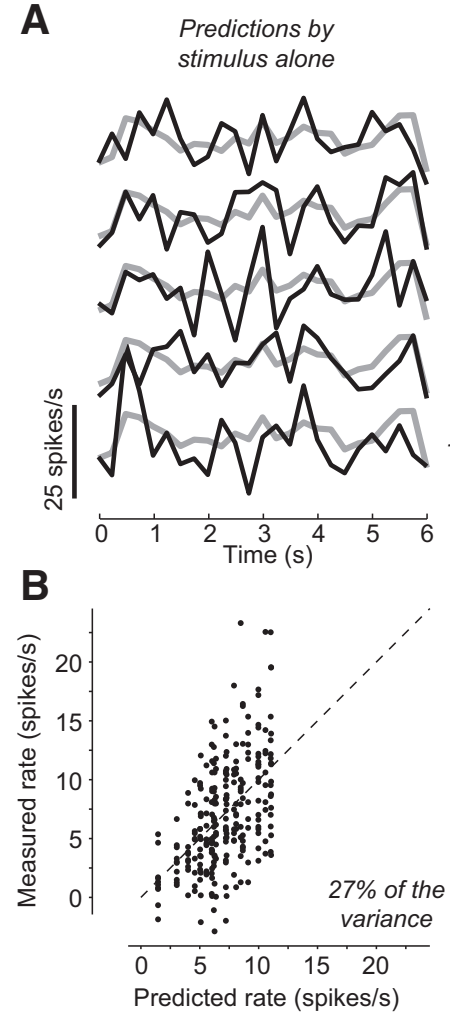

C

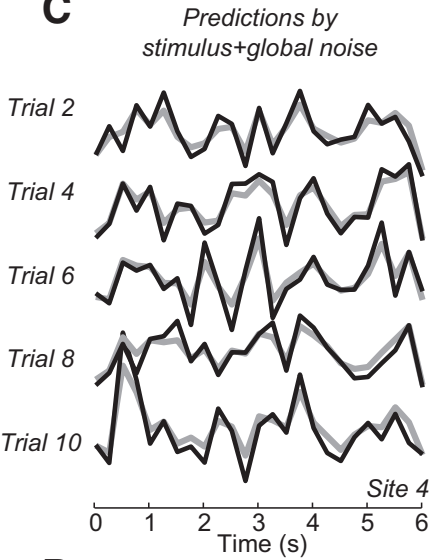

D

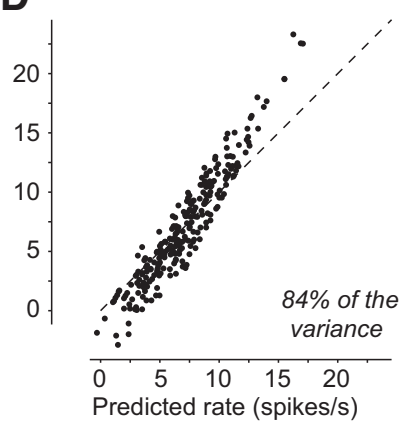

E
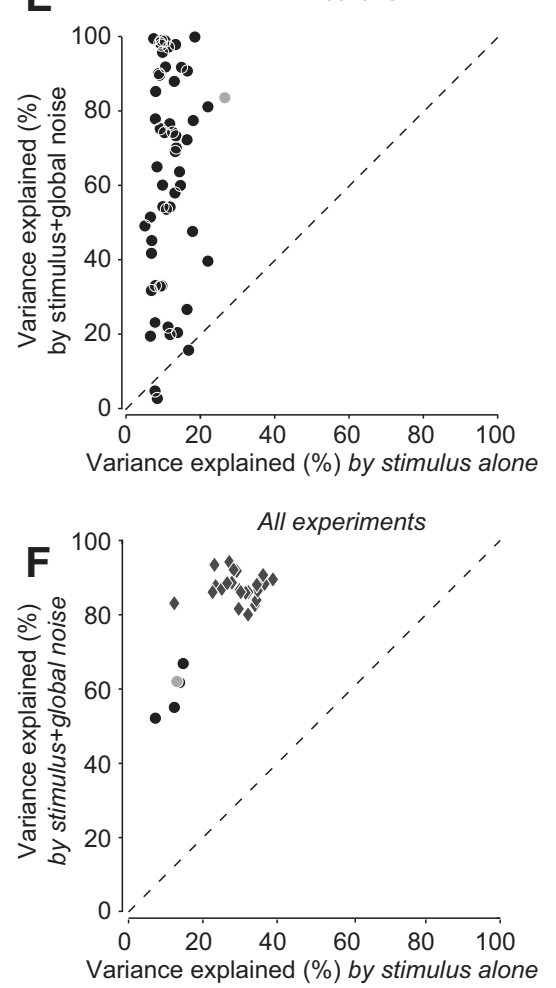

Figure 2. Predicting the variability of individual V1 neurons. A, Individual (gray lines) and trial-averaged (black lines) responses of a V1 neuron to five repetitions of a sequence of rapidly flashed, oriented gratings. The trial-averaged response can be considered a prediction of the response by the stimulus alone. $\boldsymbol{B}$, Measured spike count versus spike count predicted from the stimulus alone, in $500 \mathrm{~ms}$ bins. The stimulus explained $27 \%$ of the variance in the responses. C, The prediction (black) of the individual responses (gray) improves when taking into account the weighted global noise (measured at all the remaining neurons). $\boldsymbol{D}$, This prediction explained $84 \%$ of the variance in the responses. $\boldsymbol{E}$, Explained variance by the stimulus alone and by both stimulus and global noise. Each dot represents one neuron of the experiment shown in $\boldsymbol{A}-\boldsymbol{D}$. Gray dot represents the neuron in $\boldsymbol{A}-\boldsymbol{D}$, Experiment 79-9-11. $\boldsymbol{F}$, Explained variance in 5 different experiments (circles), by the stimulus alone and by stimulus and peers. Gray circle represents the experiment in $\boldsymbol{A}-\boldsymbol{E}$. Diamonds represent the results of the same analysis in 23 array experiments where neural activity was measured as MUA at each site rather than spike-sorted to obtain single-unit activity measured from MUA at a given site, and predicted based on MUA at the other sites.

reflect the trivial advantage conferred by adding free parameters because the predictions were cross-validated. We further considered adding a threshold nonlinearity because, in single units, the spike threshold shapes the relationship between intracellular potential and firing rate, both in terms of mean (Carandini and Ferster, 2000) and of variance (Carandini, 2004). However, a threshold would not markedly improve our fits because plotting their predictions versus the actual data revealed only minor deviations from linearity (Fig. 2D).

We obtained even better results when, instead of considering well-isolated single neurons, we turned our analysis to MUA. MUA represents the summed activity of multiple neurons located near a recording site in the array. MUA presented a major advantage: it could be readily measured at more sites of the array than single-unit activity, allowing for the analysis of all 23 experiments instead of the 5 experiments for which we had sufficient numbers of well-isolated single neurons. MUA was generally more predictable than single neuron activity on the basis of the stimulus alone (Fig. 2F, abscissa, diamonds vs circles). Still, the global noise model markedly improved predictability, just as it did for single neurons (Fig. $2 F$, ordinate). The remaining analyses, therefore, concentrate on MUA because it allows us to report on our full set of experiments.

The increased predictability of MUA over well-isolated single neurons indicates that the former has less private noise. This is to be expected if the private noise of nearby neurons is indeed private (i.e., uncorrelated even across neurons near the same recording site).
In that case, summing across those neurons (i.e., recording MUA) will reduce the impact of private noise while augmenting the impact of global noise, which is shared across neurons.

Still, the success of the global noise model in predicting MUA is particularly remarkable because MUA was considerably more variable across trials than single-unit activity measured at the same sites. We measured variability in the 5 experiments for which we had both isolated single neurons and MUA, by computing the variability index (the Fano factor, i.e., the variance divided by the mean, for spike counts measured in $500 \mathrm{~ms}$ time bins) and found it to average $2.4 \pm 0.5$ for MUA, versus $1.5 \pm 0.2$ (mean $\pm \mathrm{SE}$ ) for the single neurons measured from the same array sites. The global noise model assigns this higher variability of MUA to a shared source of noise, the global noise.

\section{Variability depends on cortical state}

The degree to which noise was shared among the population varied across experiments. In some experiments, knowing the global noise improved the predictions markedly, but in others slightly less (Fig. $2 F$ ). We asked what factors might determine this difference in variability.

We began by comparing population responses on individual trials to the trial-averaged population response (Fig. $3 A-C$ ). Because stimulus orientation is a key determinant of the responses of V1 neurons and is the only attribute that varies over the course of our stimulus (Fig. 3A), we labeled the electrode sites based on their preferred orientation, producing two-dimensional repre- 

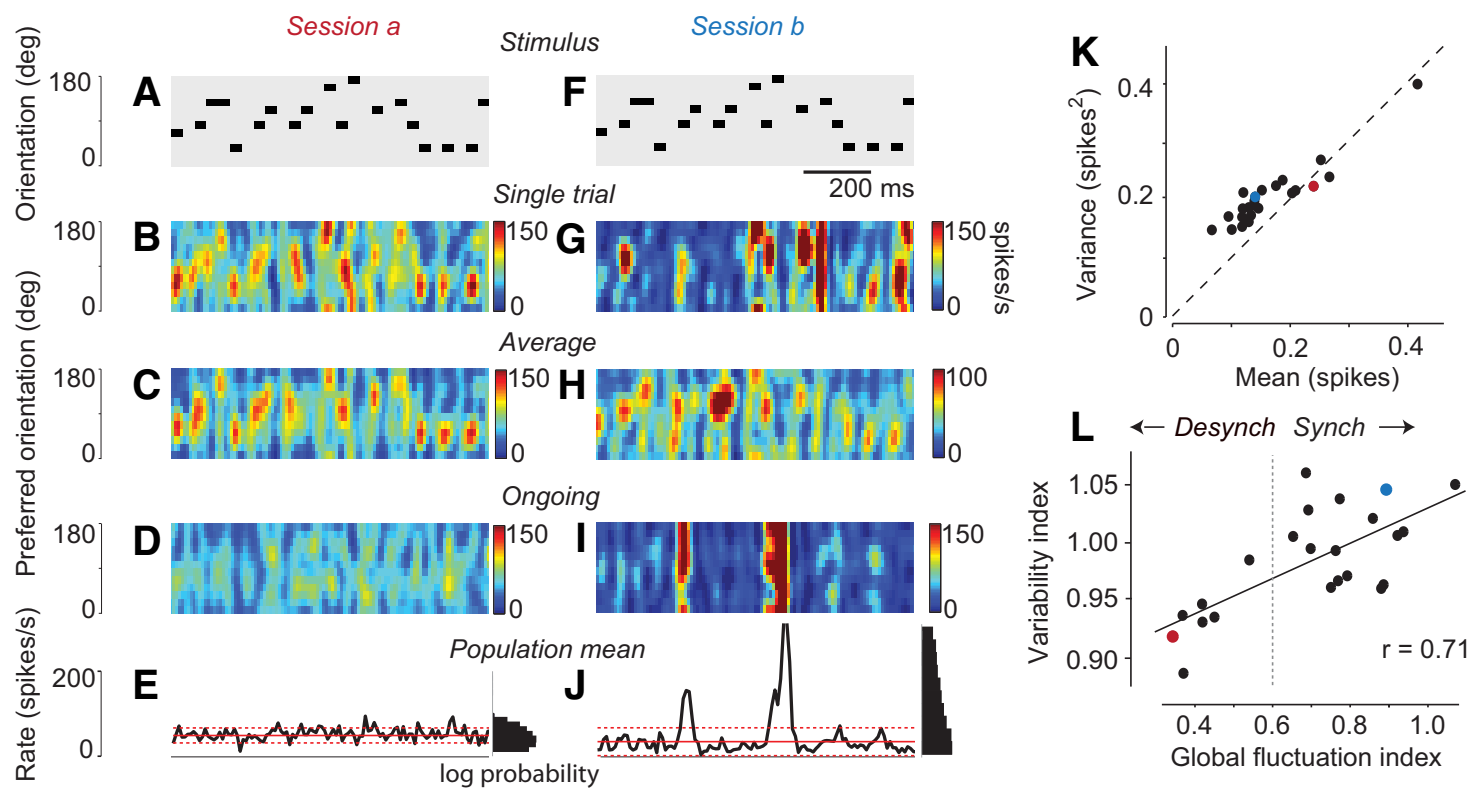

Figure 3. Variability and cortical state. $\boldsymbol{A}$, The stimulus sequence expressed as a temporal sequence of grating orientations. Only $1 \mathrm{~s}$ of stimulus is shown (stimuli typically lasted $6 \mathrm{~s}$ ). $\boldsymbol{B}$, The population response measured in one trial, as a function of time and preferred orientation. $\boldsymbol{C}$, The population response, averaged across 10 trials in this session (Experiment 79-12-3). $\boldsymbol{D}, \mathbf{A}$ single example of ongoing activity of the same population, recorded during the presentation of a blank screen during the same session. $\boldsymbol{E}$, Total population rate measured during the ongoing activity. Continuous red lines indicate average over time. Dotted red lines indicate 1 SD away from this average. Right, Histograms, Log probability of each firing rate. $\boldsymbol{F}-\boldsymbol{J}$, The same stimulus sequence was used in another experimental session (Session b) recording from the same population of neurons, $24.5 \mathrm{~h}$ earlier (Experiment 79-9-11). $\boldsymbol{K}$, Mean activity against the variance in activity for all experiments. Mean and variance were computed over $8 \mathrm{~ms}$ time bins and averaged across sites. Dots represent 23 experimental sessions in 9 animals. $A-J$, The two example experiments (recorded from the same neurons) are colored in red (Session a) and blue (Session b). $L$, Relationship between "global fluctuation index" (measured during ongoing activity) and "multiunit variability index" (measured from stimulus-driven activity) across experiments. Solid line indicates the result of linear regression. Dotted line indicates an arbitrary distinction between "synchronized" and "desynchronized" experiments. Red and blue symbols represent the two example sessions.

sentations that allowed the entire population activity to be visualized as a function of time on each trial (Benucci et al., 2009).

These graphical representations confirmed that variability differed across experimental sessions. In some sessions, cortical activity was driven reliably by the stimulus, and population responses measured in a single trial were similar to those averaged across trials (e.g., Fig. 3A-C). In other sessions, recording from the same neuronal population with the same stimuli, responses on a single trial showed isolated events involving a large portion of the population, whose occurrence seemed unrelated to the stimulus sequence (Fig. $3 F, G$ ). Indeed, these events were markedly reduced in the average across trials, which faithfully followed the changes in stimulus orientation (Fig. $3 H$ ), and resembled those of the other example session (Fig. 3C).

To extend these observations across all experiments, we defined a simple variability index as the Fano factor (variance divided by mean response) computed per site and then averaged over all sites in the experimental session, using bins of size $8 \mathrm{~ms}$. Because the mean and variance of each site were approximately similar (Fig. $3 K$ ), the variability index tended to be $\sim 1$, as expected for this bin size (Fig. 1). Nonetheless, there were clear differences across experiments, and the index ranged from 0.89 to 1.07 in our 23 experimental sessions ( 0.92 and 1.05 for the two sessions in Fig. 3A-J).

We next asked how the variability in sensory responses was related to the presence of these large, isolated events. In experiments with the highest variability, the large fluctuations in population activity were seen not only during stimulus presentations, but also in ongoing activity, recorded while showing a uniform gray screen (e.g., Fig. $3 I, J$ ). Conversely, these large events in ongoing activity were absent in experiments with low variability (e.g., Fig. $3 D, E$ ).
The large global fluctuations observed in both ongoing activity and sensory responses represent the synchronized activity of a large number of cortical neurons. They therefore resemble the "up phases" commonly observed in synchronized cortical states, such as found in sleep or deep anesthesia (Harris and Thiele, 2011).

The two examples we have seen suggest that the amount of variability seen in sensory responses is related to the size of fluctuations in spontaneous periods during the same experiment. To verify this relationship, we obtained a global fluctuation index by considering spontaneous activity and dividing the SD of the firing rate of the entire population by its mean firing rate across time.

The global fluctuation index correlated clearly with the variability index measured during sensory stimulation $(r=0.71, p=$ 0.0002 ; Fig. $3 L$ ). This correlation is not trivial because the global fluctuation index is assessed purely from ongoing activity (measured while showing a uniform gray screen), whereas the variability index is obtained in response to visual stimuli. In summary, in experiments where total activity fluctuated more strongly in the absence of any stimuli, the MUA driven by stimuli was also more variable. This relationship suggests that the same sources of variability may be at play during ongoing activity and during visually driven activity.

Within individual animals, the global fluctuation index was largely constant over the course of a single experiment (5-12 min) but varied over longer timescales (hours). These slower changes may have reflected variations in the depth of anesthesia, possibly associated with our daily injections of atropine sulfate (aimed at reducing secretions that could have blocked the air passages), or spontaneous changes in cortical state as have been described in rats (Clement et al., 2008). To guide the remaining analyses, we therefore denoted the experiments where the global fluctuation index is above a fixed criterion value as "synchronized" 

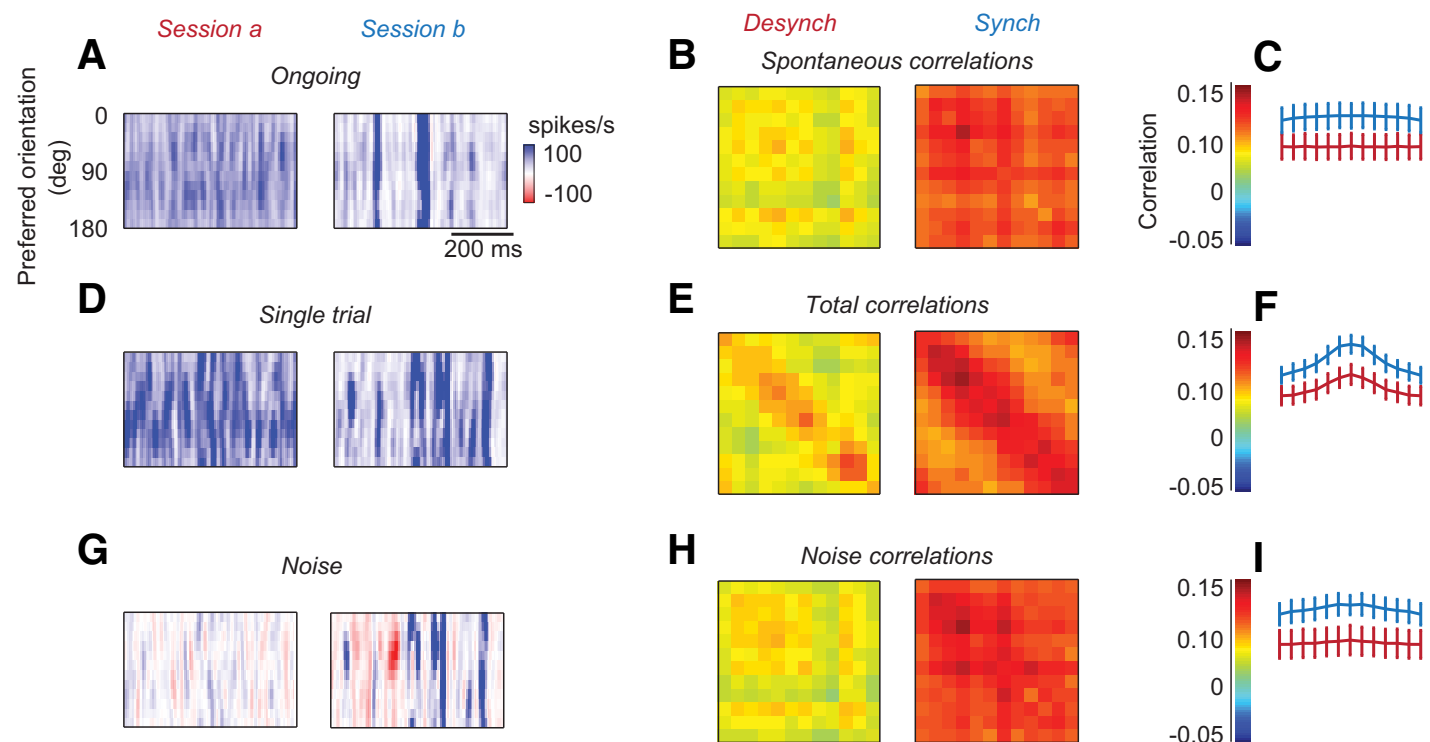

H
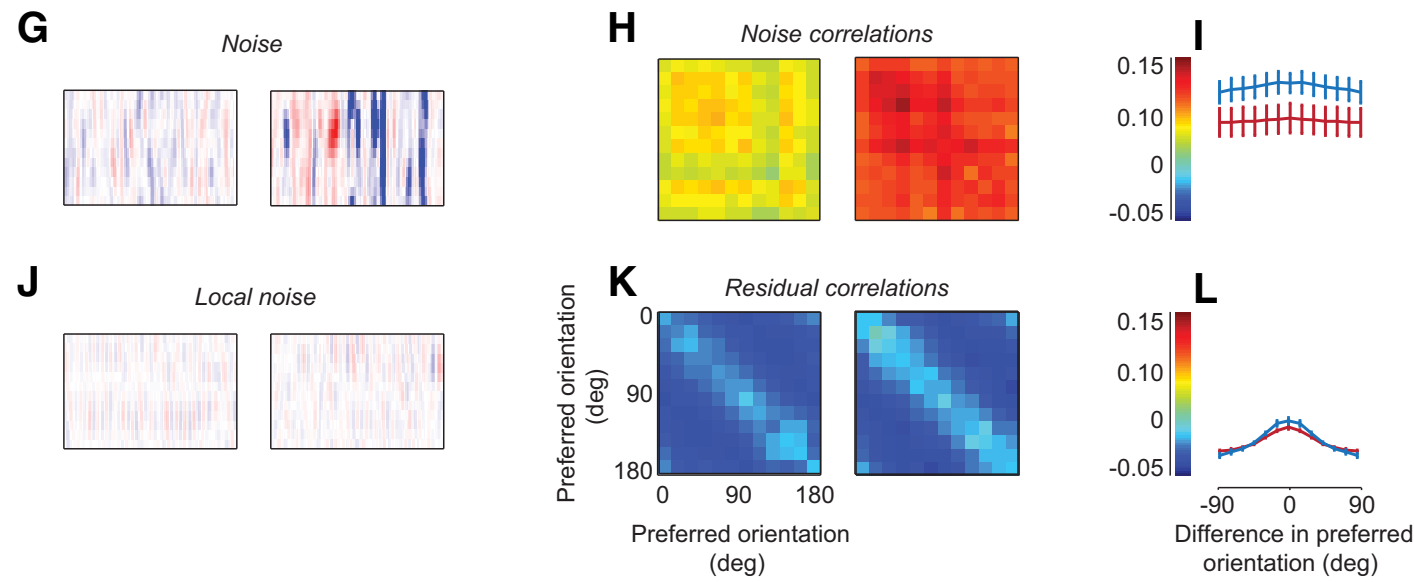

Figure 4. Cross-correlations as a function of cortical state. $\boldsymbol{A}$, Ongoing activity for two example experiments, in a desynchronized state (left) and in a synchronized state (right) (same example sessions as Fig. 3). Experiments 79-12-3 and 79-9-11. In this figure, spike trains are binned in 8 ms windows. B, Cross-correlations in activity between sites as a function of their preferred orientations, for experiments made in the desynchronized state (left) and synchronized state (right). These are known as "spontaneous correlations." $\boldsymbol{C}$, Summary of the cross-correlations in $\boldsymbol{B}$, as a function of difference in preferred orientation. Experiments were made in the desynchronized state (red) and in the synchronized state (blue). $\boldsymbol{D}-\boldsymbol{F}$, Same as $\boldsymbol{A}-\boldsymbol{C}$, for responses measured in single trials. $\boldsymbol{E}$, Correlations are known as "total correlations." $\mathbf{G}-\boldsymbol{I}$, Same as $\boldsymbol{D}-\boldsymbol{F}$, for the "noise," the difference between single trial responses and mean responses across trials. $\boldsymbol{G}$, Different color scale from $\boldsymbol{A}$ because now values can be negative. $\boldsymbol{H}$, Correlations are known as "noise correlations." $\boldsymbol{J}-\boldsymbol{L}$, Same as $\boldsymbol{G}-\boldsymbol{I}$, for the local noise: the measured noise minus the predictions of the global noise model. $\boldsymbol{K}$, Correlations are known as "residual correlations."

and the remaining ones as "desynchronized." As a criterion, we chose the value of 0.6 , as it approximately divides the experiments in two halves (Fig. 3L). However, the apparent bimodality in the global fluctuation index was not supported by statistical tests (Hartigan dip test, $p=0.077$ ). The remaining analyses, indeed, do not depend strictly on the choice of this criterion.

\section{Effects of cortical state on pairwise correlations}

The previous observations indicate that an important source of variability is provided by large population fluctuations that are present spontaneously and that are more prevalent in synchronized cortical states. The presence of such population fluctuations mathematically implies that there should be high correlations between neurons or recording sites. Specifically, a simple expression relates global fluctuations to correlations between the firing rates $R_{j}$ and $R_{k}$ of pairs of sites $j$ and $k$ (Renart et al., 2010; Harris and Thiele, 2011):

$$
\operatorname{Var}\left(\sum_{j} R_{j}\right)=\sum_{j} \operatorname{Var}\left(R_{j}\right)+\sum_{j \neq k} \operatorname{Corr}\left(R_{j}, R_{k}\right) \sqrt{\operatorname{Var}\left(R_{j}\right) \operatorname{Var}\left(R_{k}\right)}
$$

The left-hand side of this equation is the variance in population rate; the first term on the right is the summed variance of individual sites, and the third is a weighted average of pairwise correlations between sites. Because the second term on the righthand side is a sum over $O\left(N^{2}\right)$ terms whereas the first is a sum over $O(N)$ terms, the second term will typically dominate. Thus, this expression shows that the degree of global fluctuation (the left-hand side) is approximately equal to a weighted sum of pairwise correlations between individual recording sites.

We therefore next asked how pairwise correlations were affected by cortical state (Fig. 4). We considered the MUA recorded at pairs of sites in the array, computed cross-correlations between these activities, and averaged the results according to the preferred orientations of the sites, to obtain a matrix of correlations for all pairs of orientations.

First, we considered the activity measured in the absence of stimuli (uniform gray screen) and the corresponding "spontaneous correlations" (Fig. 4A-C). Experiments performed in synchronized and desynchronized states showed clear differences in the amount of spontaneous fluctuation (Fig. 4A). The prevalence of fluctuations affected the pairwise correlations between sites, which were higher in the synchronized than desynchronized cortex (Fig. 4B). Notably, these correlations showed a very weak dependence on the orientations preferences of the recording sites, as expected from correlations that were caused by fluctuations investing the overall population simultaneously (Fig. 4C).

We then turned to the stimulus responses and the corresponding "total correlations." As expected, these correlations showed a strong dependence on orientation similarity, as they 
included the "signal correlations" that are driven by stimulus responses themselves. In addition, however, activity in synchronized states contained spontaneous global fluctuations (Fig. 4D), which led to higher correlations across sites (Fig. 4E). Correlations were uniformly higher in the synchronized cortex, with no apparent influence of the difference in preferred orientation (Fig. $4 F)$. This effect of state was seen also in recordings performed hours apart within a single experiment, confirming that cortical state is an important determinant of correlations.

Similar results were obtained when we examined the trial-bytrial fluctuations in the population and the correlated variability, or "noise correlation," between sites (Fig. $4 G-I$ ). Noise is the difference between activity measured in a trial and activity averaged across trials. As expected, it was more prominent in the more synchronized experiment (Fig. 4G). Indeed, noise correlations were lower in the desynchronized than in the synchronized cortex (Fig. 4H). These effects were common in all recordings (Fig. 4I). Once again, only a weak dependence of correlation on orientation similarity was seen (Fig. 4I), although it appeared slightly larger than during spontaneous activity (Fig. 4C).

These data indicate that cortical state has a marked influence on pairwise correlations, accounting for much of their strength. Pairwise correlations are uniformly higher in synchronized states than in desynchronized states, whether they are measured during spontaneous activity (Fig. $4 B, C$ ), during visual responses (Fig. $4 E, F)$, or in the trial-by-trial deviations from the mean responses (Fig. 4H,I).

This marked difference in pairwise correlations across states could be largely explained by a single factor: global fluctuations that invest the whole population (Fig. 4J-L). To verify this impression, we measured the local noise (Fig. $4 J$ ) by subtracting the weighted global noise (Fig. 2C) from the noise measured on each trial (Fig. $4 G$ ). In other words, we ran the same global noise model that we had run earlier (Fig. 2), except that we now ran it with a time bin of $8 \mathrm{~ms}$ rather than $500 \mathrm{~ms}$, and we considered the residuals of this model. The resulting local noise (Fig. $4 J$ ) was generally small compared with the measured noise (Fig. $4 G$ ). In other words, the global noise provided a good estimate of the noise at each site, just as we had seen at the slower time scales (Fig. 2). Importantly, the local noise had a similar magnitude whether it came from measurements performed in desynchronized or synchronized states (Fig. $4 J$ ). Indeed, subtracting the global noise completely removed the differences between pairwise correlations measured in the desynchronized and synchronized states (Fig. $4 K$ ), making the two have similar amplitudes (Fig. 4L). We conclude that the higher variability and pairwise correlations found in the synchronized state are both due to the presence of large global activity fluctuations.

These large global fluctuations tended to invest all neurons, regardless of their orientation preference. Indeed, unlike what would have been expected based on prior studies using voltagesensitive dye imaging (Tsodyks et al., 1999; Kenet et al., 2003), correlations in ongoing activity were not measurably higher between sites of similar preferred orientation (Fig. 4C). Neither did the noise correlations show such an orientation dependence (Fig. $4 H, I)$. By contrast, the matrix of total correlations showed a clear diagonal structure (Fig. 4E,F), which was fully expected because the stimulus concurrently drives the neurons that have similar orientation preference (Fig. 4D). The absence of this structure from ongoing activity and noise activity confirms that the large activity fluctuations, which drove correlations and were stronger during synchronized states, tended to invest the overall population simultaneously.

\section{Discussion}

Because its sensory responses are relatively well understood, area V1 has long served as a workbench to investigate trial-to-trial variability. Here we have asked to what extent V1 neurons are more variable than LGN neurons, and we have sought to understand their variability from the standpoint of large V1 populations. By relating the variability at one cortical site to that in the LGN and at other cortical sites, we were able to argue that variability in V1 reflects global, population-wide fluctuations, which do not favor neurons based on their preferred orientation and which are strongest in synchronized states. These global fluctuations markedly increase pairwise correlations and fully explain how these correlations depend on cortical state.

Consistent with previous studies that compared variability in LGN and in V1 (Kara et al., 2000; Goris et al., 2014), we found that cortical variability was higher in individual V1 neurons than in individual LGN neurons. This observation agrees with previous estimates of variability in LGN (for review, see Sadagopan and Ferster, 2012) and in V1 (for review, see Carandini, 2004).

One possible factor contributing to the increased variability observed in V1 is the pooling of LGN inputs. If variability were highly correlated among LGN neurons, it would become larger once V1 pools their activity. Indeed, Sadagopan and Ferster (2012) showed that LGN neurons can have strongly correlated variability if their receptive fields are aligned. Moreover, they reported that silencing inputs from a local region of cortex leaves the variability of a number of V1 neurons unchanged. Nevertheless, many other V1 cells did reduce variability following silencing of local V1 inputs. We speculate that these differences between neurons may have reflected differing cortical states during the experiments of Sadagopan and Ferster (2012).

Our findings show that cortical state is an important determinant of response variability in V1. High variability was coupled to the occurrence of large spontaneous fluctuations in activity that did not depend on orientation preference. Such fluctuations indicate a more synchronized cortical state. Work in somatosensory, auditory, and visual cortex has shown that responses to sensory stimuli can vary widely depending on cortical state (Kisley and Gerstein, 1999; Poulet and Petersen, 2008; Curto et al., 2009; Marguet and Harris, 2011), and that also the variability in those responses is subject to cortical state; for instance, variability in auditory cortex is highest when ongoing cortical activity exhibits rhythmic population bursts (Kisley and Gerstein, 1999). Also, variability in rat visual cortex (and in LGN) decreases upon activation of the nucleus basalis (Goard and Dan, 2009), which presumably induces a more active and desynchronized cortical state.

Our findings highlight the determinant role of cortical state on pairwise correlations. It has long been recognized that correlations do not typically reflect direct connections between neurons (Moore et al., 1970); they are more often interpreted as reflecting the fraction of shared inputs (Ts'o et al., 1986; Alonso et al., 2001; Ko et al., 2011). Our data, by contrast, show that correlations in cat $\mathrm{V} 1$ can vary from moment to moment, as a function of cortical state. This observation agrees with previous results. For instance, in rat visual cortex, overall levels of spontaneous correlation increase as state transitions from wakefulness to anesthesia (Greenberg et al., 2008). In rat auditory cortex, mean correlations may reduce close to zero in desynchronized states (Renart et al., 2010). Finally, in monkey visual cortex, large activity fluctuations and noise correlations vary depending on state (Ecker et al., 2010, 2014). These findings indicate that correlations do not simply reflect anatomical circuit features but arise 
dynamically in the network. Indeed, mean pairwise correlations can be close to zero even in strongly and densely connected circuits (Renart et al., 2010).

More generally, these results confirm the view that population activity structure depends on brain state and that pairwise correlations are often a reflection of activity synchronized at the level of the population (Harris and Thiele, 2011; Okun et al., 2012). Indeed, in our global noise model, the sole source of variability and correlation in cat $\mathrm{V} 1$ are the events that synchronously invest the overall population. Such correlations have previously been interpreted as large-scale fluctuations in mean excitability, which can lead to correlated spiking behavior (Brody, 1998). Which common input drives these mean excitability fluctuations remains to be seen. The differing occurrence of these events between cortical states was sufficient to explain the differences in correlation and variability between these states. Furthermore, such a strong dependence of pairwise correlations on cortical state might explain many of the differences in correlation values observed across studies (Cohen and Kohn, 2011).

Because our measurements were all conducted under anesthesia, we cannot say whether the variations in cortical state that we encountered would occur during wakefulness. It would be of interest to repeat the measurements in awake animals, but it is not common to record from awake cats. Moreover, our measurements were performed in a preparation (anesthetized cat V1) that has given rise to a vast literature, which still guides leading views of neocortical function. In contrast to previous work in this same preparation using voltage imaging (Tsodyks et al., 1999; Kenet et al., 2003), we found no evidence for orientation-related structure in spontaneous population activity and little evidence for structure in the variability of population sensory responses. Indeed, our measurements indicate that spontaneous fluctuations in firing rate tended to invest all neurons regardless of preferred orientation and little tendency for spontaneous correlations to be higher between neurons with similar orientation preference. It remains to be determined whether there exist sources of structured noise that are not aligned to the dimension that we considered, orientation preference.

We defined cortical state in terms of the size of spontaneous fluctuations in MUA data, rather than from the more traditionally used power spectra of LFP or EEG signals. The two measurements are strongly related, as LFP and EEG fluctuations correlate with both instantaneous population firing rates and intracellular potentials (Poulet and Petersen, 2008; Curto et al., 2009; Okun et al., 2010; Saleem et al., 2010). Studies in rat cortex have shown that the definition based on MUA provides more fine-grained measurements of state than that based on LFP power spectra (Curto et al., 2009). This said, it would have been interesting to relate cortical state in the recorded population to overall brain state as recorded elsewhere.

Some dependence on preferred orientation could be seen in noise correlations and, more markedly, in the correlations of the local noise. The orientation dependence of noise correlations is consistent with earlier reports (Bair et al., 2001; Kohn and Smith, 2005; Smith and Kohn, 2008; Ch'ng and Reid, 2010; Cohen and Kohn, 2011). However, the selectivity of noise correlations was extremely mild. Rather than the difference in orientation preference between two sites, the primary determinant of noise correlations in our study was clearly cortical state, similar to recent findings in monkey cortex (Ecker et al., 2014). Indeed, when we removed the effect of global noise, the pairwise correlations became practically independent of cortical state (Fig. $5 F$ ). Cortical state, therefore, is a prime determinant of correlation strength, similarly to other known factors, such as distance, firing rate, and cortical layer (Cohen and Kohn, 2011). The orientation preference seen in the local noise, finally, is likely to be the result of a nonadditive interaction between signal and noise, and we are currently pursuing the possibility that it might be multiplicative (Goris et al., 2014).

Together, our data indicate that the variability of $\mathrm{V} 1$ population responses to stimuli is mainly caused by spontaneously generated activity that is highly correlated across the neuronal population. This view of area V1 agrees with results obtained in primate area MT (Buracas et al., 1998), and in rat auditory cortex (Deweese and Zador, 2004), where a substantial portion of the variability of responses in single neurons can be predicted by knowledge of the nearby local field potential.

Cortical state, in turn, determines how prevalent these fluctuations are and thus acts as a key determinant of the variability seen in V1 sensory responses and in ongoing activity. A limitation of our study is that the variations in cortical state were due to causes outside our control, such as fluctuations in the depth of anesthesia. Nonetheless, these fluctuations allowed us to probe an effect of cortical state that could be present also in the awake brain as it engages or disengages from a task (Harris and Thiele, 2011; Tan et al., 2014). By providing a clearer picture of variability and by showing that this variability is strongly determined by cortical state, our results may help elucidate the aspects of visual responses that are not directly attributable to visual stimuli.

\section{References}

Alonso JM, Usrey WM, Reid RC (2001) Rules of connectivity between geniculate cells and simple cells in cat primary visual cortex. J Neurosci 21:4002-4015. Medline

Arieli A, Sterkin A, Grinvald A, Aertsen A (1996) Dynamics of ongoing activity: explanation of the large variability in evoked cortical responses. Science 273:1868-1871. CrossRef Medline

Bair W, Zohary E, Newsome WT (2001) Correlated firing in macaque visual area MT: time scales and relationship to behavior. J Neurosci 21:16761697. Medline

Benucci A, Ringach DL, Carandini M (2009) Coding of stimulus sequences by population responses in visual cortex. Nat Neurosci 12:1317-1324. CrossRef Medline

Bonin V (2005) Contrast gain control, noise, and spike threshold in the early visual system. PhD Thesis. Zurich: Swiss Federal Institute of Technology.

Bonin V, Mante V, Carandini M (2003) Predicting signal and noise in LGN and V1. Program No. 229.5. In: Online Abstract Viewer. Washington, DC: Society for Neuroscience.

Bonin V, Mante V, Carandini M (2005) The suppressive field of neurons in lateral geniculate nucleus. J Neurosci 25:10844-10856. CrossRef Medline

Bradley A, Skottun BC, Ohzawa I, Sclar G, Freeman RD (1987) Visual orientation and spatial frequency discrimination: a comparison of single neurons and behavior. J Neurophysiol 57:755-772. Medline

Brody CD (1998) Slow covariations in neuronal resting potentials can lead to artefactually fast cross-correlations in their spike trains. J Neurophysiol 80:3345-3351. Medline

Buracas GT, Zador AM, DeWeese MR, Albright TD (1998) Efficient discrimination of temporal patterns by motion-sensitive neurons in primate visual cortex. Neuron 20:959-969. CrossRef Medline

Carandini M (2004) Amplification of trial-to-trial response variability by neurons in visual cortex. PLoS Biol 2:E264. CrossRef Medline

Carandini M, Ferster D (2000) Membrane potential and firing rate in cat primary visual cortex. J Neurosci 20:470-484. Medline

Ch'ng YH, Reid RC (2010) Cellular imaging of visual cortex reveals the spatial and functional organization of spontaneous activity. Front Integr Neurosci 4:pii20. CrossRef Medline

Clement EA, Richard A, Thwaites M, Ailon J, Peters S, Dickson CT (2008) Cyclic and sleep-like spontaneous alternations of brain state under urethane anaesthesia. PLoS One 3:e2004. CrossRef Medline

Cohen MR, Kohn A (2011) Measuring and interpreting neuronal correlations. Nat Neurosci 14:811-819. CrossRef Medline 
Cohen MR, Maunsell JH (2009) Attention improves performance primarily by reducing interneuronal correlations. Nat Neurosci 12:1594-1600. CrossRef Medline

Curto C, Sakata S, Marguet S, Itskov V, Harris KD (2009) A simple model of cortical dynamics explains variability and state dependence of sensory responses in urethane-anesthetized auditory cortex. J Neurosci 29: 10600-10612. CrossRef Medline

Dean AF (1981) The variability of discharge of simple cells in the cat striate cortex. Exp Brain Res 44:437-440. Medline

Deweese MR, Zador AM (2004) Shared and private variability in the auditory cortex. J Neurophysiol 92:1840-1855. CrossRef Medline

Dinstein I, Heeger DJ, Lorenzi L, Minshew NJ, Malach R, Behrmann M (2012) Unreliable evoked responses in autism. Neuron 75:981-991. CrossRef Medline

Durand S, Freeman TC, Carandini M (2007) Temporal properties of surround suppression in cat primary visual cortex. Vis Neurosci 24:679-690. CrossRef Medline

Ecker AS, Berens P, Keliris GA, Bethge M, Logothetis NK, Tolias AS (2010) Decorrelated neuronal firing in cortical microcircuits. Science 327:584587. CrossRef Medline

Ecker AS, Berens P, Cotton RJ, Subramaniyan M, Denfield GH, Cadwell CR, Smirnakis SM, Bethge M, Tolias AS (2014) State dependence of noise correlations in macaque primary visual cortex. Neuron 82:235-248. CrossRef Medline

Geisler WS, Albrecht DG (1997) Visual cortex neurons in monkeys and cats: detection, discrimination, and identification. Vis Neurosci 14:897-919. CrossRef Medline

Goard M, Dan Y (2009) Basal forebrain activation enhances cortical coding of natural scenes. Nat Neurosci 12:1444-1449. CrossRef Medline

Goris RL, Movshon JA, Simoncelli EP (2014) Partitioning neuronal variability. Nat Neurosci 17:858-865. CrossRef Medline

Greenberg DS, Houweling AR, Kerr JN (2008) Population imaging of ongoing neuronal activity in the visual cortex of awake rats. Nat Neurosci 11:749-751. CrossRef Medline

Harris KD, Thiele A (2011) Cortical state and attention. Nat Rev Neurosci 12:509-523. CrossRef Medline

Harris KD, Csicsvari J, Hirase H, Dragoi G, Buzsáki G (2003) Organization of cell assemblies in the hippocampus. Nature 424:552-556. CrossRef Medline

Heggelund P, Albus K (1978) Response variability and orientation discrimination of single cells in striate cortex of cat. Exp Brain Res 32:197-211. Medline

Kara P, Reinagel P, Reid RC (2000) Low response variability in simultaneously recorded retinal, thalamic, and cortical neurons. Neuron 27:635646. CrossRef Medline

Kenet T, Bibitchkov D, Tsodyks M, Grinvald A, Arieli A (2003) Spontaneously emerging cortical representations of visual attributes. Nature 425 : 954-956. CrossRef Medline

Kisley MA, Gerstein GL (1999) Trial-to-trial variability and statedependent modulation of auditory-evoked responses in cortex. J Neurosci 19:10451-10460. Medline

Ko H, Hofer SB, Pichler B, Buchanan KA, Sjöstrom PJ, Mrsic-Flogel TD (2011) Functional specificity of local synaptic connections in neocortical networks. Nature 473:87-91. CrossRef Medline

Kohn A, Smith MA (2005) Stimulus dependence of neuronal correlation in primary visual cortex of the macaque. J Neurosci 25:3661-3673. CrossRef Medline

Kohn A, Zandvakili A, Smith MA (2009) Correlations and brain states: from electrophysiology to functional imaging. Curr Opin Neurobiol 19:434438. CrossRef Medline

Levine MW, Troy JB (1986) The variability of the maintained discharge of cat dorsal lateral geniculate cells. J Physiol 375:339-359. Medline

Mainen ZF, Sejnowski TJ (1995) Reliability of spike timing in neocortical neurons. Science 268:1503-1506. CrossRef Medline

Mante V, Bonin V, Carandini M (2008) Functional mechanisms shaping lateral geniculate responses to artificial and natural stimuli. Neuron 58: 625-638. CrossRef Medline
Marguet SL, Harris KD (2011) State-dependent representation of amplitude-modulated noise stimuli in rat auditory cortex. J Neurosci 31:6414-6420. CrossRef Medline

Moore GP, Segundo JP, Perkel DH, Levitan H (1970) Statistical signs of synaptic interaction in neurons. Biophys J 10:876-900. CrossRef Medline

Nauhaus I, Benucci A, Carandini M, Ringach DL (2008) Neuronal selectivity and local map structure in visual cortex. Neuron 57:673-679. CrossRef Medline

Okun M, Naim A, Lampl I (2010) The subthreshold relation between cortical local field potential and neuronal firing unveiled by intracellular recordings in awake rats. J Neurosci 30:4440-4448. CrossRef Medline

Okun M, Yger P, Marguet SL, Gerard-Mercier F, Benucci A, Katzner S, Busse L, Carandini M, Harris KD (2012) Population rate dynamics and multineuron firing patterns in sensory cortex. J Neurosci 32:17108-17119. CrossRef Medline

Pillow JW, Shlens J, Paninski L, Sher A, Litke AM, Chichilnisky EJ, Simoncelli EP (2008) Spatio-temporal correlations and visual signalling in a complete neuronal population. Nature 454:995-999. CrossRef Medline

Poulet JF, Petersen CC (2008) Internal brain state regulates membrane potential synchrony in barrel cortex of behaving mice. Nature 454:881-885. CrossRef Medline

Priebe NJ, Ferster D (2012) Mechanisms of neuronal computation in mammalian visual cortex. Neuron 75:194-208. CrossRef Medline

Reich DS, Victor JD, Knight BW, Ozaki T, Kaplan E (1997) Response variability and timing precision of neuronal spike trains in vivo. J Neurophysiol 77:2836-2841. Medline

Renart A, de la Rocha J, Bartho P, Hollender L, Parga N, Reyes A, Harris KD (2010) The asynchronous state in cortical circuits. Science 327:587-590. CrossRef Medline

Ringach DL (2009) Spontaneous and driven cortical activity: implications for computation. Curr Opin Neurobiol 19:439-444. CrossRef Medline

Ringach DL, Hawken MJ, Shapley R (1997) Dynamics of orientation tuning in macaque primary visual cortex. Nature 387:281-284. CrossRef Medline

Sadagopan S, Ferster D (2012) Feedforward origins of response variability underlying contrast invariant orientation tuning in cat visual cortex. Neuron 74:911-923. CrossRef Medline

Saleem AB, Chadderton P, Apergis-Schoute J, Harris KD, Schultz SR (2010) Methods for predicting cortical UP and DOWN states from the phase of deep layer local field potentials. J Comput Neurosci 29:49-62. CrossRef Medline

Shadlen MN, Newsome WT (1998) The variable discharge of cortical neurons: implications for connectivity, computation, and information coding. J Neurosci 18:3870-3896. Medline

Smith MA, Kohn A (2008) Spatial and temporal scales of neuronal correlation in primary visual cortex. J Neurosci 28:12591-12603. CrossRef Medline

Steriade M (2006) Grouping of brain rhythms in corticothalamic systems. Neuroscience 137:1087-1106. CrossRef Medline

Tan AY, Chen Y, Scholl B, Seidemann E, Priebe NJ (2014) Sensory stimulation shifts visual cortex from synchronous to asynchronous states. Nature 509:226-229. CrossRef Medline

Tolhurst DJ, Movshon JA, Dean AF (1983) The statistical reliability of signals in single neurons in cat and monkey visual cortex. Vision Res 23:775785. CrossRef Medline

Truccolo W, Hochberg LR, Donoghue JP (2010) Collective dynamics in human and monkey sensorimotor cortex: predicting single neuron spikes. Nat Neurosci 13:105-111. CrossRef Medline

Ts'o DY, Gilbert CD, Wiesel TN (1986) Relationships between horizontal interactions and functional architecture in cat striate cortex as revealed by cross-correlation analysis. J Neurosci 6:1160-1170. Medline

Tsodyks M, Kenet T, Grinvald A, Arieli A (1999) Linking spontaneous activity of single cortical neurons and the underlying functional architecture. Science 286:1943-1946. CrossRef Medline

Vogels R, Spileers W, Orban GA (1989) The response variability of striate cortical neurons in the behaving monkey. Exp Brain Res 77:432-436. CrossRef Medline 